\title{
Hybrid Micro Textures - A Multi-Scale Surface Engineering Approach For Enhancing Tribological Characteristics
}

\author{
S Niketh and G L Samuel \\ Department of Mechanical Engineering, Indian Institute of Technology Madras, India \\ samuelgl@iitm.ac.in
}

\begin{abstract}
Surface engineering techniques have already been reported to be one of the fascinating areas of research in the manufacturing industries due to its uniqueness in enhancing the tribological characteristics. Design and development of optimal microscale textures at the tribological interfaces can minimize the sliding friction, which will eventually result in the improved part performance and life. Among the various surface engineering techniques, laser micromachining principle has got wide acceptance owing to its capability in generating microfeatures of any intricate shapes with high processing speed and precise geometrical quality. Hence in the present work, Nd: YAG laser was used to create hybrid microscale textures on the disc surfaces and the same were used for the tribology experiments for evaluating the effectiveness of hybrid textures in enhancing the frictional characteristics. Tribological experiments showed a significant reduction of $32.65 \%$ in friction coefficient for hybrid textured surfaces than the smooth surfaces. The outcomes of present work demonstrate the feasibility of applying hybrid microscale textures at the contact regimes of mechanical components like bearings, mechanical seal, etc. and also on cutting tools for achieving better productivity.
\end{abstract}

DOI: $10.2961 /$ jlmn.2019.03.0006

Keywords: tribology, laser, texturing, friction, asperity, interface

\section{Introduction}

\subsection{Surface engineering for tribology enhancement}

Surface engineering techniques are now one of the fascinating areas in the manufacturing research world as it can bring enhanced performances of mechanical systems in terms of energy conservations as well as reliability. Implementation of sustainable designs and surface modifications for mechanical components can results in prolonged part life, thereby minimizing the maintenance and replacement cost Hamilton, Walowit and Allen [1]. Surface functionalization techniques span a wide range of processes which involves deposition/removal of materials, whose dimensions vary from nanometer to millimeter scale. Material deposition techniques comprise mainly of physical vapor deposition (PVD), chemical vapor deposition (CVD), anodizing, laser processing, and cold spraying methods [2]. For achieving a high-quality coating, greater knowledge about the chemical composition and coating parameters are required for accomplishing the desired performances. This creates difficulty in applying coating techniques over a wide spectrum.

Surface functionalization techniques are employed in the manufacturing industries to improve tribological characteristics and thereby to minimize the chance of mechanical failures of components. Friction and wear arising at the sliding interface of mechanical units are reported to be the main source initiating the failure of mechanical components and parts [3]. This motivated the tribologists to explore the mechanism responsible for the occurrence of friction and wear and to find an alternative to prevent sliding friction. Researchers have studied various possibilities in friction control by applying surface coatings, adding lubricants, cryogenic cooling, etc. Even though these techniques are successful in friction reduction, they are reported to be effective for a particular application under certain conditions. Hence there is always a need to find an alternative technique which can be implemented in the mechanical components to minimize energy loss resulting from the sliding friction and thereby to enhance the product performance.

Coating of surfaces which are having intricate geometries will be highly challenging for accomplishing uniform coating thickness [4]. Moreover, the coating life highly depends on the operating temperature of the component, which will have a direct impact on the total production cost [5]. The present work focuses on minimizing the frictional heating generating on cutting tool, especially on drill tools while machining. Apart from the complex tool geometry, as machining happens inside the hole, frictional heating will be more severe. This can be solved by creating microscale textures, which will act as micro reservoirs of cutting fluid, which will increase the cooling efficiency compared to coated tools [6]. The enhancement in tribological characteristics will also further depends upon the texture geometry and its distribution [7]. Zenebe Segu and Hwang [8] experimentally proved the enhancement in tribological characteristics by implementing multi-scale textures on the contact surfaces. Hence the same concept of multi-scale textures has been followed in the present work and is demonstrated in terms of the pin on disc experiments under variable conditions of sliding velocity and sliding environment (dry and wet). 


\subsection{Micromachining - Principle and applications}

Micro-manufacturing technology has emerged as an advanced machining concept which plays a vital role in the manufacturing world for developing miniaturized features/components whose dimension varies from submillimeter to nanometer range. Various fields including automobile, aerospace, biomedical, spacecraft, etc. have been already reported to be benefitted from the principle of micromachining in terms of reduction in fuel consumption along with extended life of the products/components. Scaling down in the product dimension can bring significant benefits in terms of both economy and energy conservation. Now a day, with the emergence of ultra-precision machining techniques, a significant increment in the application of micro-components can be seen in the area of microelectronics, optoelectronics, biosensors, etc. [9]. The principle of micro-manufacturing in a broader way can be explained as the integration of numerous factors, which includes manufacturing methods (traditional or non-traditional), precision and ultra-precision machines, and organizational planning and machining strategies.

Micromachining techniques include both conventional and unconventional based machining processes, which includes LIGA, laser machining, focused ion beam, micro-electro discharge grinding, electroplating, micro stereolithography, etc. [10]. Micro electro discharge machining technique has already been accepted in the manufacturing industries, including aerospace, automobile, spacecraft, etc. as it is capable of creating complex-shaped parts. Rashed et al., 2014 [11] experimentally proved the applicability of Micro EDM in generating quality micro holes for the application of fuel injector nozzles. They reported a net reduction of $32 \%$ in surface roughness with respect to conventional manufacturing processes. In another work Rashed et al., 2013 [12] reported a novel approach of combining water jet guided laser as an alternative for the micro EDM for creating higher qualitative fuel injector nozzle. Their experiments showed an average $R_{q}$ of $450 \mathrm{~nm}$ for the surface generated using EDM, whereas a significant reduction in the roughness of $150 \mathrm{~nm}$ was reported in case of surface processed by Laser micro-jet technique. Apart from EDM, ultrasonic micromachining (UMM) technique are also widely used to create microfeature even on difficult to machine materials like titanium alloy [13]. Electrochemical micromachining (ECMM) technique are also widely used in creating micro features with controlled geometry. Recently, Patel et al., 2019 [14] showed the applicability of ECMM in creating microscale textures on flat and curved surfaces.

Among all the micromachining techniques, due to higher lateral resolution by minimized focusability, the laser micromachining technology is proved to be the most qualified technique which can generate micro-features of any intricate geometries with higher dimensional accuracy [15-17]. More recently, femtosecond lasers have captured the attention of micromachining industries due to its higher capability in producing micro features with minimum surface defects [18-21]. Femtosecond lasers can ablate any surfaces with lesser heat affected zone [22, 23]. The key characteristic principle behind this phenomenon is due to the material interaction of high-intensity laser pulses in a short time frame $[24,25]$.

\subsection{Surface texturing of mechanical components}

Micro-texturing of mechanical components is one of the well-established techniques which can enhance tribological characteristics of mechanical components. Design and development of micro/nanoscale specific patterned textures on the sliding surfaces have already reported being efficient in controlling the propagation of surface wear. Adhesion and deformation mechanisms occurring at the microscopic regime of asperity contact interfaces are responsible for wear transmission due to ploughing phenomenon resulting from the interlocking effect of micro asperities under the application of normal load. Researchers and manufacturers have applied various micromachining techniques in developing periodic surface patterns (surface texturing) on the sliding surfaces to improve tribological characteristics of the contact boundaries. Functionalization of surfaces by creating micro features can results in many advantageous factors, which includes improvement in load carrying capacity, wear resistance, friction coefficient, etc. [7]. (Jeng, Y. R., 1996) [8] has applied the principle of surface texturing for the first time in the area of cylinder liner honing, and was also reported to be the first commercial application of textured surfaces. Later it was applied on magnetic storage devices and MEMS devices for overcoming adhesion and stiction [9]. The effectiveness of microtextures in enhancing the tribological characteristics greatly depends on its geometric quality, which includes its bottom shape and separation from adjacent micro asperities [10]. Fundamental research work on various forms and shapes of surface textures has been carried out by several research groups worldwide, and it was reported that the optimum geometrical features differ from one area of application to another. Hamilton et al. (1966) [11] had done surface texturing in the form of micro asperities (dimples) on the bearings and found to be effective in generating additional hydrodynamic pressure, thereby improving the bearing life. Etsion and Burstein (1996) [12] developed a model for micro dimpled mechanical seals and proved to be effective in improving the seal performance while comparing with the non-textured seal surfaces. Friction and wear reduction while using microtextured surfaces is attributed to various underlying mechanisms which involve wear debris entrapment, rise in hydrodynamic lubrication pressure, and reduction in contact length. This was proved by Joshi et al., 2018 [26] by conducting tribological experiments on microtextured surfaces using femtosecond laser micromachining under lubricated non-conformal point contact. Nowadays, femtosecond laser micro texturing is widely used due to lesser surface defects. Recently Pimenov et al., 2019 [27] used femtosecond laser surface texturing to create diamond-like nanocomposite film for the application of friction reduction. They reported the reliability of ultra-short laser source in generating controlled features with variable area density, which played a predominant role in friction reduction.

Though the effect of microscale textures on tribological characteristics are well analyzed both experimentally and analytically in many literatures, there is high dearth in research work exploring the effect of hybrid scale microtextures in frictional properties. This forms the prime objective of the present work which involves, (i) to create hybrid microscale textures by laser micromachining pro- 
cesses, (ii) to evaluate its effectiveness in enhancing the tribological characteristics and (iii) application on the cutting tool having free form surfaces like drill tool.

\section{Experimental details}

\subsection{Laser processing of test specimen}

Neodymium-doped Yttrium aluminum garnet laser having a wavelength of $1064 \mathrm{~nm}$ was used with a repetition rate of $2.5 \mathrm{kHz}$ and pulse duration of $20 \mathrm{~ns}$. Micro textures of variable geometry were created on the carbide disc having $50 \mathrm{~mm}$ diameter and $10 \mathrm{~mm}$ thickness. Whereas, Grade 5 titanium alloy (Ti-6Al-4V) was used as the pin material, which acted as the counter sliding surface for the rotating disc. The diameter of the pin was chosen to be $10 \mathrm{~mm}$ having a height of $35 \mathrm{~mm}$. Figure 1 represents the schematic sketch of the laser processing unit and Figure 2 shows the orientation of micro features with respect to normal load applied on the pin. For understanding the effectiveness of various microfeature shapes on the tribological characteristics, in the present study, different test specimens having variable feature shapes were employed. Table 1 shows the details of test specimens used for the tribological evaluation. Area density was calculated based on the imaginary unit cell occupying the micro features on the test specimen.

Table 1 Details of tribology experiments

\begin{tabular}{lll}
\hline TY PE & DESIGN & \multicolumn{1}{c}{ SPECIFICA TION } \\
\hline Dimple & & $\begin{array}{l}\text { Diameter- } 90 \mu \mathrm{m} \\
\text { Pitch }-135 \mu \mathrm{m}\end{array}$ \\
Depth $-60 \mu \mathrm{m}$ \\
Area density- $34.88 \%$
\end{tabular}

Furthermore, to investigate the performance of microtextures under various lubrication regimes, tribology experiments were carried under dry and wet sliding regime conditions. Under all conditions of tribo tests, the effectiveness of microtextures was assessed in terms of the coefficient of friction.

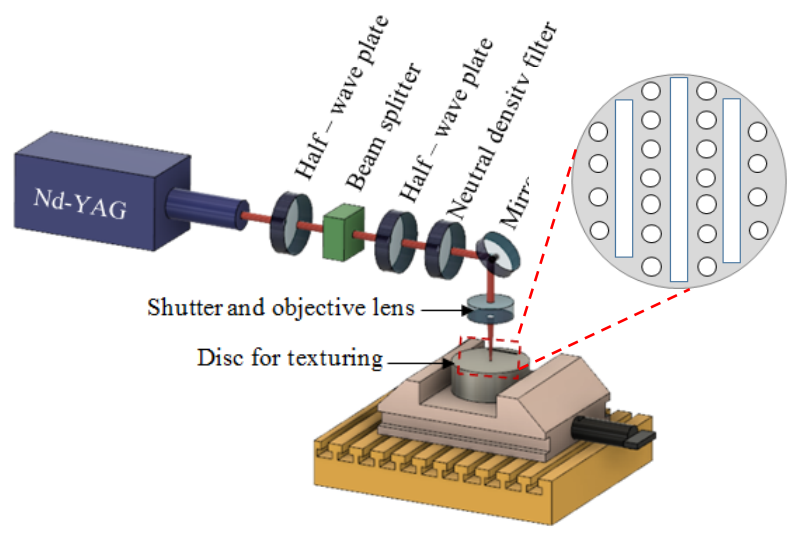

Fig. 1 Schematic sketch of laser micro texturing

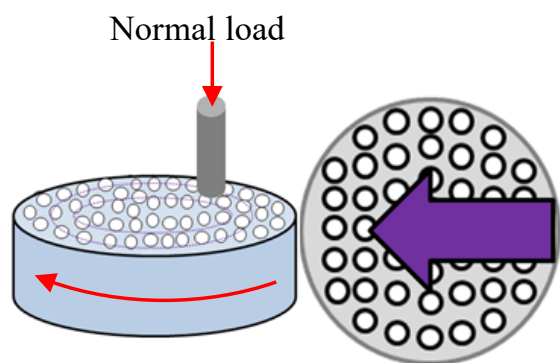

(a). Dimple textured surface

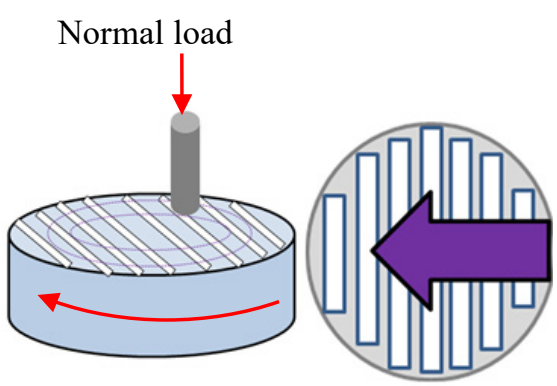

(b). Groove textured surface

Normal load

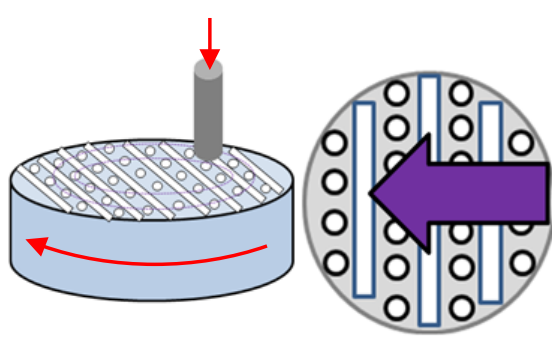

(c). Hybrid textured surface

Fig. 2 Details of sliding regime under various conditions 
Figure 2 represents the schematic representation of the sliding regime of various samples during tribological experiments. Under all experimental conditions, the pin was kept stationary where a normal load is applied against rotating disc. The grooves are aligned perpendicular to the sliding direction..

\subsection{Characterization of microtextures}

For ensuring the feature shape and geometrical accuracy, the laser textured microfeatures on the test samples were analyzed using stereomicroscope and 3D dimensional profilometer. Figure 3 shows the stereomicroscopic image of the test samples having dimple, groove, and hybrid textures. From the microscopic analysis, it has been observed that the geometrical quality of the textures was greatly affected by the formation of recast layer owing to the unevenness in heat distribution over the target surface. All the test specimen was grounded and polished using the diamond paste to remove the burrs around the feature boundary. Moreover, all the samples were cleaned using ultrasonic bath using acetone to remove further entrapped dust particles before conducting tribology tests.

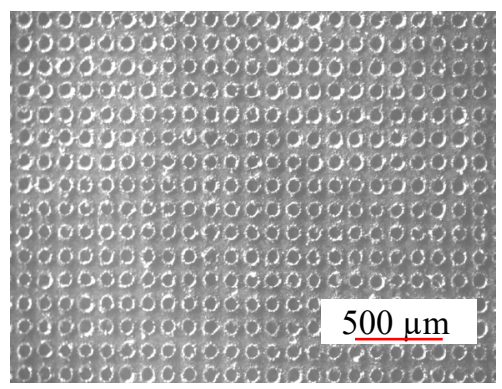

(a) Micro dimple surface

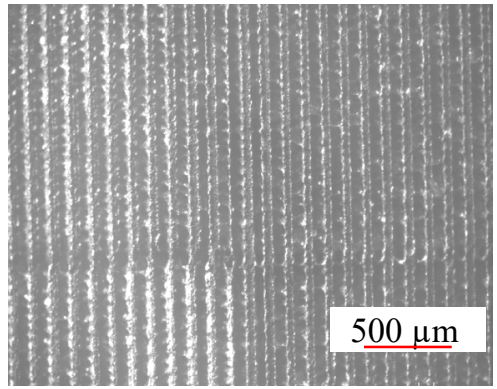

(b) Micro grooved surface

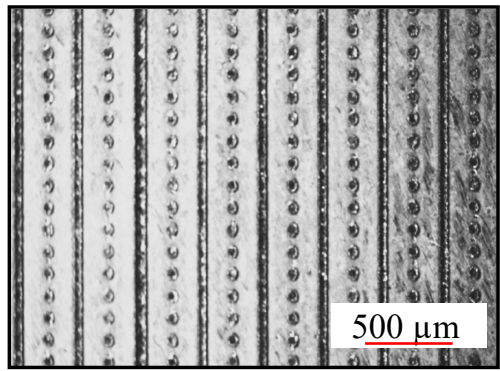

(c) Hybrid surface

Fig. 3 Stereomicroscopic image of textured surfaces

Figure 4 (a) represents the three-dimensional representation of the micro dimpled surface before cleaning where the burr height was about $25 \mu \mathrm{m}$ which reduced to $4.2 \mu \mathrm{m}$ after polishing process as shown in Figure 4 (b). The bottom shape of the dimples was observed to be in the form of hemispherical shape. The diameter of dimples was observed to be greatly affected by the re-solidification layer. Hence for assuring a common measuring procedure for calculating the depth, in the present paper, the depths were taken from the top of the test specimen to the lowest surface of the dimple. The same procedure is followed for all the samples in the present study. Furthermore, as the diameter was affected by the uneven melting of the surface, inner diameter was taken for further analysis, as mentioned in Figure 5.

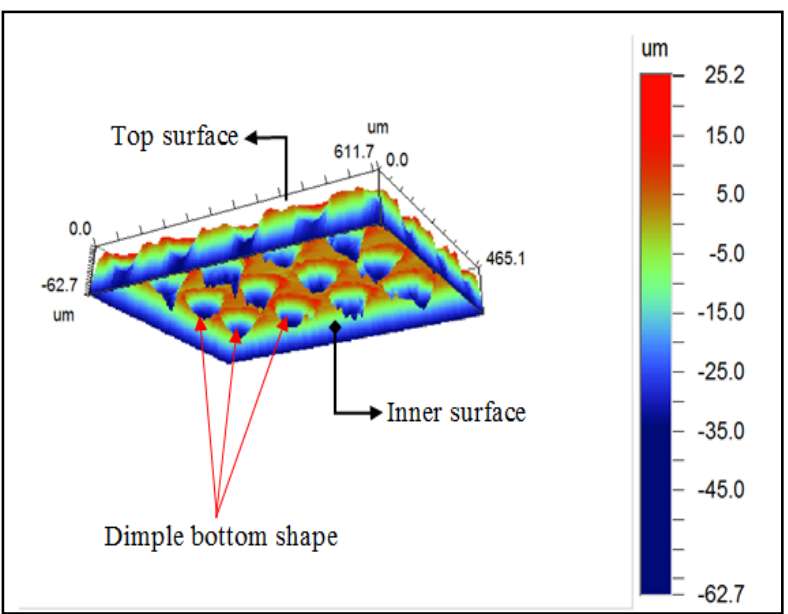

(a) Before polishing

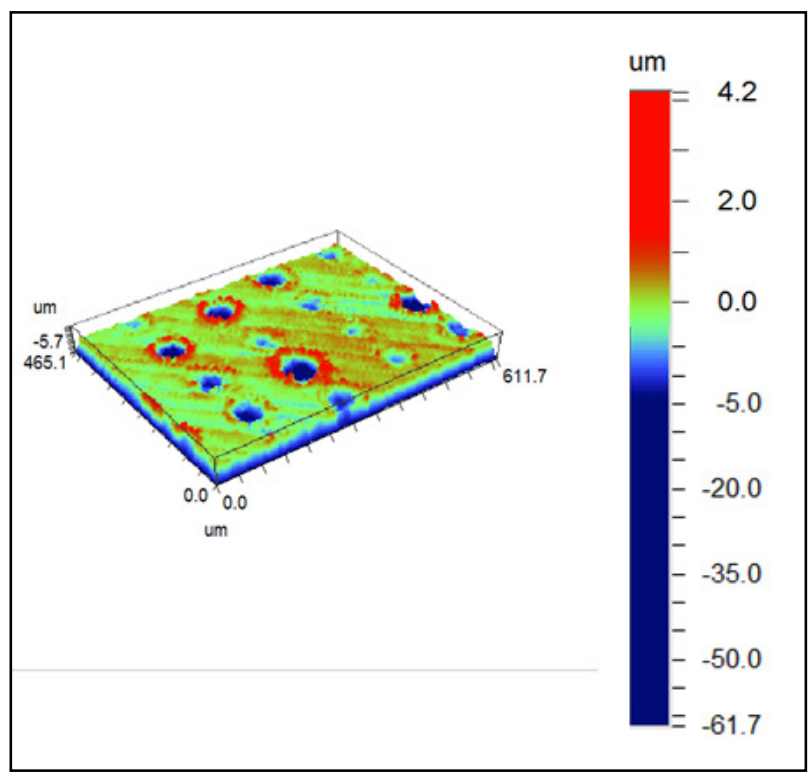

(b) After post processing

Fig.4 Characterization of dimpled surface 


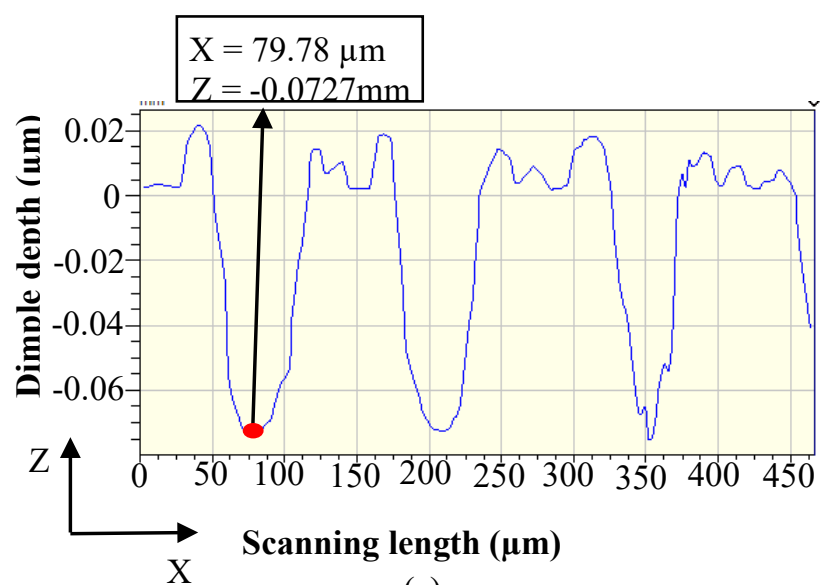

(a)

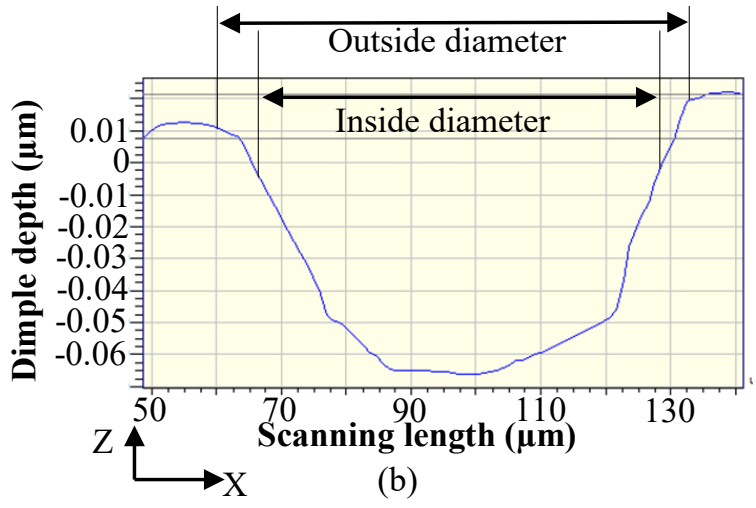

Fig. 5 (a) Profile of micro dimples, (b) Details of depth measurement of single dimple

From the characterization of laser processed microfeatures, a high level of non-uniformity in the feature shape and depth was observed. This can be attributed to the poor beam quality and also the unevenness in heat distribution on the material surface during laser interaction [28]. As the microfeatures in the present work were created using a nanosecond laser, material ejection phenomenon will be more dominant than vaporization, affecting the feature geometry and shape. This can be further understood by analyzing the interaction of laser pulses with the material subsurface layer. The physical mechanism occurring during the interaction of laser pulses with the ceramic material can be categorized as (i) reflection, (ii) absorption, (iii) scattering and (iv) transmission [29]. Among these, the occurrence of multiple reflectivities is reported to be the main factor affecting the depth profile of the machined features on the work material $[30,31]$. Apart from this, the absorptivity of the material also plays a predominant role in determining the quality of the microfeature. Chryssolouris [32] reported that for better absorption, the angle of incidence between the laser pulses and work material has to be more than $80^{\circ}$. The abovementioned problems can be resolved using a femtosecond laser, where the material ablation (photoablation) takes place by direct vaporization, which eventually results in the formation of microfeature of higher geometrical quality [33]. This can be the scope for extension of the present work.

\subsection{Tribological evaluation}

Friction tests were performed using a pin on a disc set up where the pin made up of Titanium alloy (Ti-6Al-4V) was kept stationary against a rotating disc made up of Tungsten Carbide. Furthermore, to investigate the perfor- mance of micro-textured surfaces under various lubrication regimes, tribology experiments were also carried under various sliding regime conditions, including dry and wet. It should be noted that under all conditions of tribotests, the effectiveness of microscale textures was evaluated mainly based on the formation of the coefficient of friction. For understanding the effect of sliding speed on frictional behavior, the sliding speed was varied from $0.50 \mathrm{~m} / \mathrm{s}$ to $1 \mathrm{~m} / \mathrm{s}$. Table 2 depicts the specifications of the tribological experiments.

Table 2 Details of pin on disc test

\begin{tabular}{ccc}
\hline & Disc & Pin \\
\hline Material & $\begin{array}{c}\text { Tungsten } \\
\text { carbide }\end{array}$ & $\begin{array}{c}\text { Titanium alloy - } \\
\text { Grade } 5\end{array}$ \\
Diameter $(\mathrm{mm})$ & 50 & 10 \\
Height $(\mathrm{mm})$ & 10 & 35 \\
\hline \multicolumn{3}{c}{ Test conditions }
\end{tabular}

Sliding condition - Dry, Wet

Disc velocity $(\mathrm{m} / \mathrm{s})-0.50,0.67,0.83,1$

\section{Results and discussions \\ 3.1 Coefficient of friction}

Tribology experiments revealed a better reduction in the frictional effect for the test samples having microscale textures. Under the dry condition, the coefficient of friction was observed to be higher (0.49) for the plain surface. Coefficient of friction was found to be reduced to 0.38 for the surface with hybrid textures, showing a net reduction of $22.45 \%$ in friction was obtained while using hybrid textured surfaces, which substantiated the friction reduction property of micro-textured surfaces when applied at the sliding contact regimes. Figure 6 shows the comparison of the variation of the coefficient of friction with respect to sliding time for various test samples under dry condition.

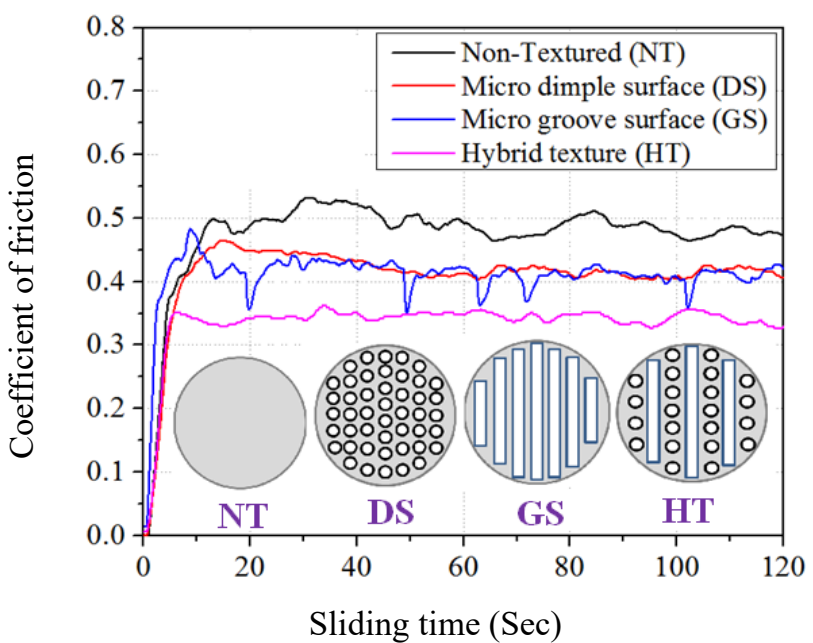

Fig. 6 Variation of coefficient of friction with sliding time under dry condition at sliding speed of $1 \mathrm{~m} / \mathrm{s}$

In the present work, a higher reduction in sliding friction was observed under the wet condition when compared to the dry tribology experiments. Under wet condition also, 
the surfaces with hybrid textures were reported to be performed better than all other test samples recording a lower coefficient of friction of 0.23 . Higher coefficient of friction value, 0.38 was recorded for the plain surface, which got reduced to 0.29 for micro dimpled surface and 0.26 for the micro-grooved surface. This shows the lubrication enhancing phenomenon of microtextures due to the formation of cavitation pressure resulting in hydrodynamic lubrication. Figure 7 represents the comparison of the coefficient of friction for various test samples under different lubrication regimes. The improved performance of hybrid textured surfaces can be related to the fundamental property of the surface texturing principle which can be explained in terms of contact length reduction, entrapment of wear debris and the formation of hydrodynamic lubrication [34-38]. At the sliding interface, the interaction of micro asperity will always result in the formation of wear debris formation due to the ploughing effect resulting from the interlocking of micro-asperities on the counter sliding surfaces. The surfaces with micro textures can entrap these wear particles, thereby eliminating the third body interaction between the sliding regimes. Moreover, the microtextures will act as the micro pool which can retain the lubricants thereby improving the fluid film thickness leading to better hydrodynamic pressure, which will eventually, transforms the mode of contact of sliding pairs from boundary lubrication to hydrodynamic lubrication. Whereas, under dry sliding condition owing to the higher heat generation, adherence of the counter sliding surfaces takes place, leading to the cold welding of micro asperities which will make the surface wear to propagate rapidly. As the microtextures can minimize contact length of sliding surfaces, surface adhesion can be minimized, which will be eventually beneficial in the reduction of frictional effect. Figure 8 represents the mechanism of micro-textured surfaces under the various sliding regime.

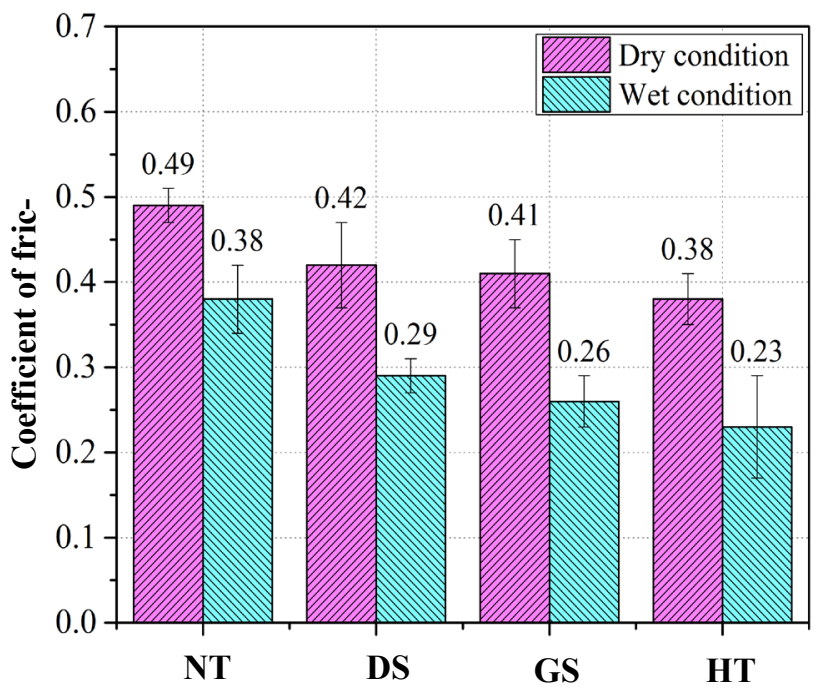

Fig. 7 Coefficient of friction under various conditions at sliding speed of $1 \mathrm{~m} / \mathrm{s}$

Figure 9 depicts the variation of the coefficient of friction with respect to sliding speed under dry condition. As the sliding velocity is increased from $0.5 \mathrm{~m} / \mathrm{s}$ to $1 \mathrm{~m} / \mathrm{s}$, a reduction in the coefficient of friction was observed from the tribology experiments.

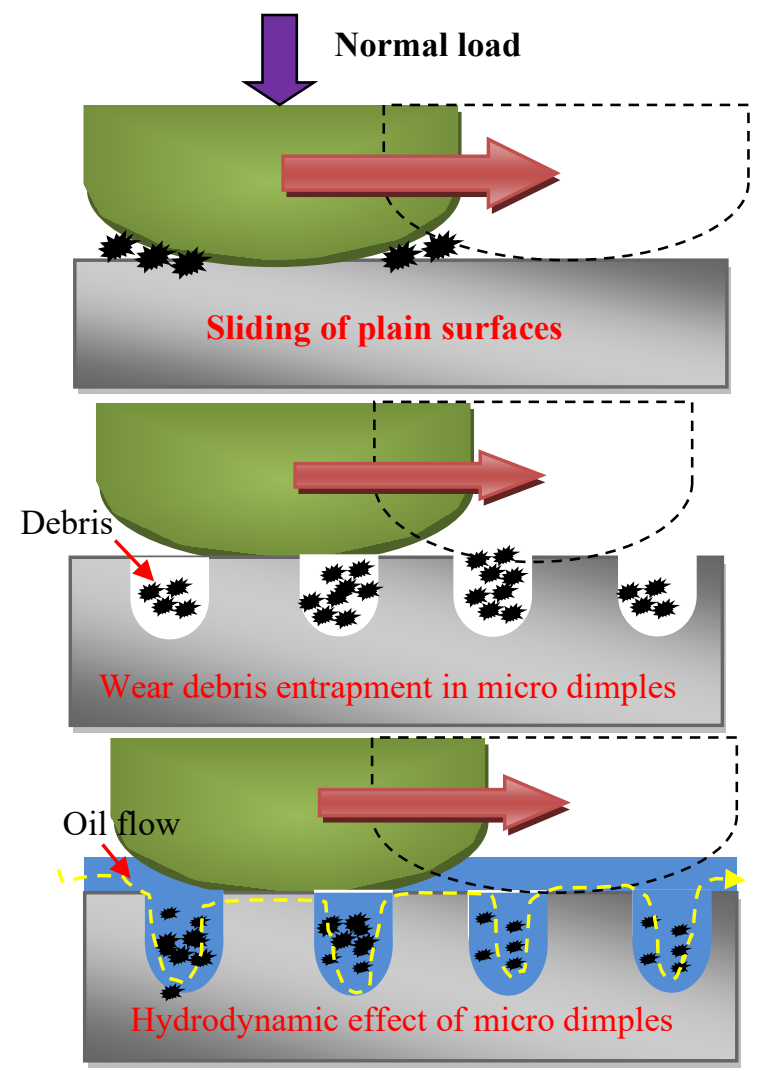

Fig. 8 Mechanism of friction reduction phenomenon

At the lower sliding speed of $0.5 \mathrm{~m} / \mathrm{s}$, the coefficient of friction was observed to be a minimum of 0.43 for the test sample having hybrid textures. Due to higher material asperity interaction resulting from adhesion and deformation mechanism coefficient of friction was found to be higher (0.54) for non-textured test sample compared to the micro dimpled surface (0.47) and micro-grooved surfaces (0.46). Hence a net reduction of $20.37 \%$ in friction coefficient was achieved for the test samples having hybrid textured surfaces. An even better result was achieved while increasing the sliding velocity from $0.5 \mathrm{~m} / \mathrm{s}$ to $1 \mathrm{~m} / \mathrm{s}$. At $1 \mathrm{~m} / \mathrm{s}$, coefficient of friction was reported to be lesser $(0.38)$ for the hybrid textured sample when compared to dimple textured surface $(0.42)$, grooved textured surface $(0.41)$ and non-textured surface (0.49).

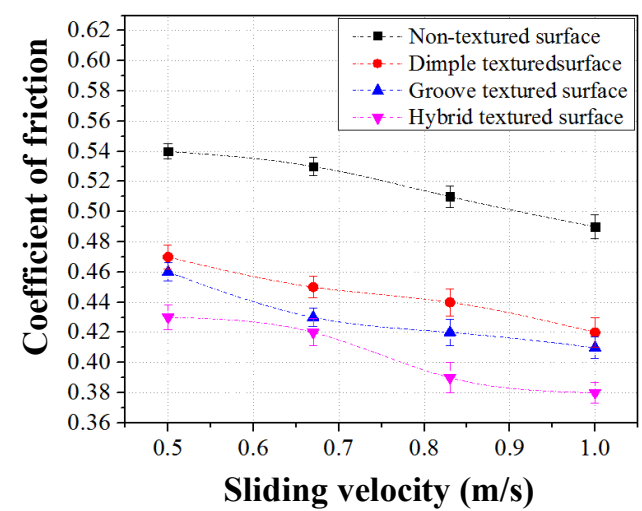

Fig. 9 Variation of coefficient of friction with respect to sliding velocity under dry condition 
Tribology experiments were also extended under wet conditions by varying the sliding velocity for unveiling the micro-texture effect in improving the lubrication effect. Under wet condition also test samples having hybrid textures were observed to be exhibit better friction reduction property which recorded a coefficient of friction of 0.3 at $0.5 \mathrm{~m} / \mathrm{s}$ and 0.23 at $1 \mathrm{~m} / \mathrm{s}$ as shown in Figure 10 . Hence a net reduction of $30.43 \%$ in the coefficient of friction was observed with the increment in sliding velocity. A similar trend was also observed in the case of test samples with micro dimples and microgrooves. For the micro dimpled surfaces, the coefficient of friction was found to be 0.35 at $0.5 \mathrm{~m} / \mathrm{s}$ and 0.29 at $1 \mathrm{~m} / \mathrm{s}$. Whereas in the case of microgrooved surfaces, the coefficient of friction got reduced from 0.32 to 0.26 with the increase in sliding velocity from $0.5 \mathrm{~m} / \mathrm{s}$ to $1 \mathrm{~m} / \mathrm{s}$. Hence the tribology results from the present work it can be concluded that there is a gradual reduction in friction coefficient with the increment in sliding velocity, which can be attributed to the transformation of hydrodynamic lubrication from the boundary lubrication as per the stribeck curve [39]. This can be further related to the formation of lubricant film thickness between the interface of two sliding surfaces separating the counter surface, which will reduce the surface wear and friction. Similar kinds of observations were also reported by many researchers $[34,35,40-44]$ were surface texturing was found to be the effective method in improving the tribological behavior which favors in the transition of the mode of surface interaction from boundary lubrication to hydrodynamic lubrication resulting from the formation of fluid film thickness. The reduction in friction coefficient with respect to sliding velocity also greatly depends on the fluid viscosity and normal load acting on the sliding pair. In the present work, though the variation sliding velocity was considered in a smaller range $(0.5 \mathrm{~m} / \mathrm{s}$ to $1 \mathrm{~m} / \mathrm{s})$, a considerable reduction in friction coefficient was observed for test samples including textured and non-textured one. An even better reduction in friction coefficient can be achieved by increasing the sliding velocity and also by providing high viscosity lubrication oil at the sliding interface, which can be the scope of extension of the present work.

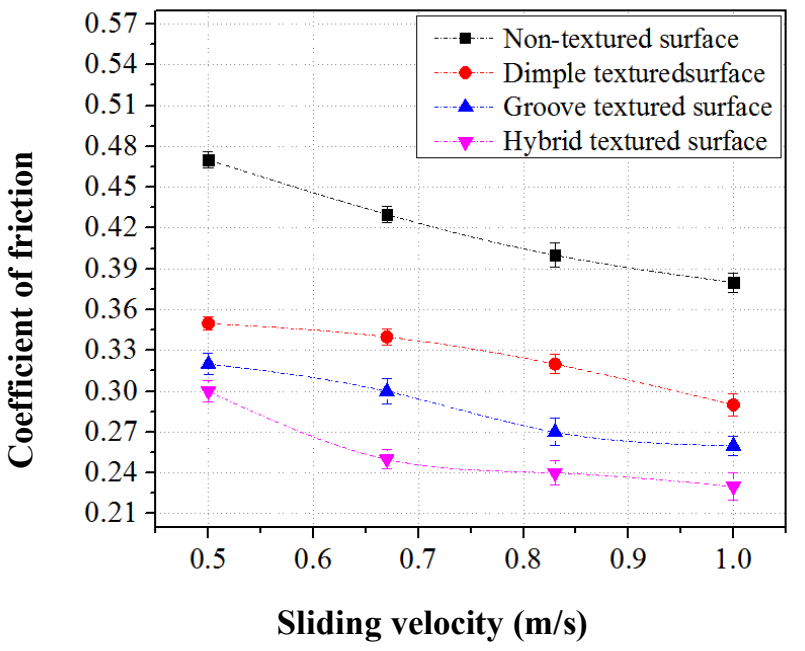

Fig. 10 Variation of coefficient of friction with respect to sliding velocity under wet condition

\subsection{Surface morphology analysis}

All test specimens were analyzed using a scanning electron microscope for understanding the morphological variations induced due to the sliding conditions. Figure 11 represents the surface details of all the test samples used in the present work.

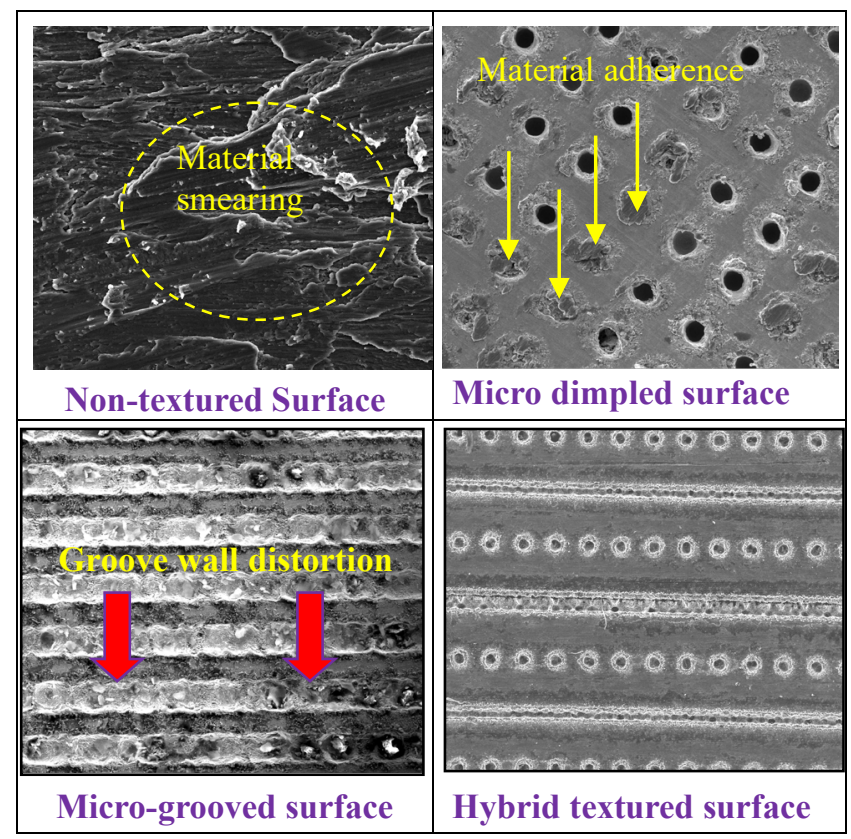

Fig. 11 Surface morphology analysis of test specimens

From the SEM image, it was observed that for the nontextured surface, a higher rate of material smearing was formed owing to the higher contact pressure resulting from the third body interaction of wear debris particles. As the microtextures were effective in entrapping this wear particle, the surface morphology of all the textured surfaces was found to be better than that of the non-textured surface. Observations at the micro-scale further reveal the distortion of the edge wall of both dimples and grooves, which reveals the occurrence of higher strain gradient resulting from the severe contact stresses. Moreover in the present work as titanium alloy was used as one of the tribo pair material, its low thermal conductivity property largely affected in the material adhesion due to the accumulation of heat at the sliding regime.

\subsection{Application of hybrid textures on drill tool}

From the tribology results, it has been understood that the hybrid microscale textures are highly beneficial in friction reduction even under dry sliding conditions. Hence an attempt has been done to apply the concept of hybrid surface texturing on drill tool for improving the machining performance. As drilling is an internal cutting process, frictional heating on the drilling surface will be more due to the sliding contact between the flute-chip interface as well as margin-hole wall interface. Creating microtextures on the drill tool surface is a real challenge owing to its complex tool geometry and free from the surface. Figure 12 shows the microscopic image of the hybrid textured drill tool. Microdimples of $90 \mu \mathrm{m}$ diameter and depth of $60 \mu \mathrm{m}$ 
were created along with microgrooves of $50 \mu \mathrm{m}$ width and depth of $60 \mu \mathrm{m}$.

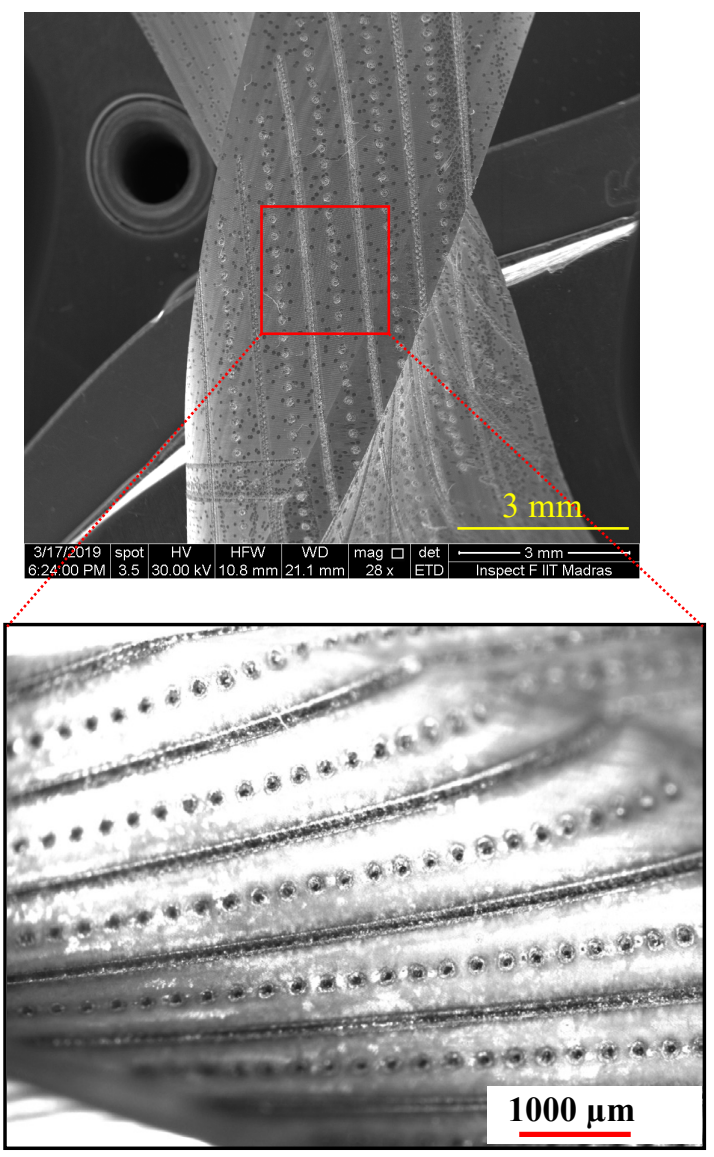

Fig. 12 Drill tool with hybrid micro scale textures

\subsection{Drilling forces while machining Ti-6Al-4V}

In the present work for understanding the effectiveness of hybrid micro scale textures in enhancing the machining performances, drilling experiments were conducted. Carbide drill tool with and without textures were used for creating through holes of $10 \mathrm{~mm}$ depth on the titanium work material (Ti-6Al-4V). Figure 13 depicts the schematic representation of the drilling experiments. The effectiveness of the hybrid micro scale textures on the drill tool were assessed mainly based on the variation in thrust force and torque. For recording the variation in drilling forces, a cutting force dynamometer (Make: Kistler) was used under all drilling conditions. Usually in drilling the cutting force profile has three stages including the initial stage where a rapid increment in cutting force occurs, which will be followed by a intermediate stage where the force profile will be almost stationary showing the maximum magnitude in cutting forces. Beyond this will be the final stage representing the disengagement of drill tool from the work material showing a sudden drop in the cutting forces. The intermediate stage usually starts after the drill point length where the tool gets fully engaged with the work material.

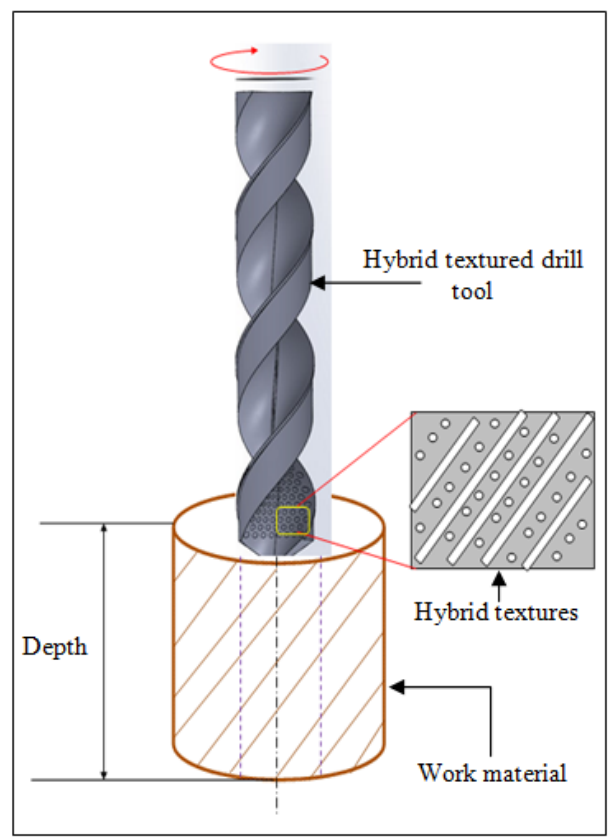

Fig. 13 Drilling using hybrid textured tool

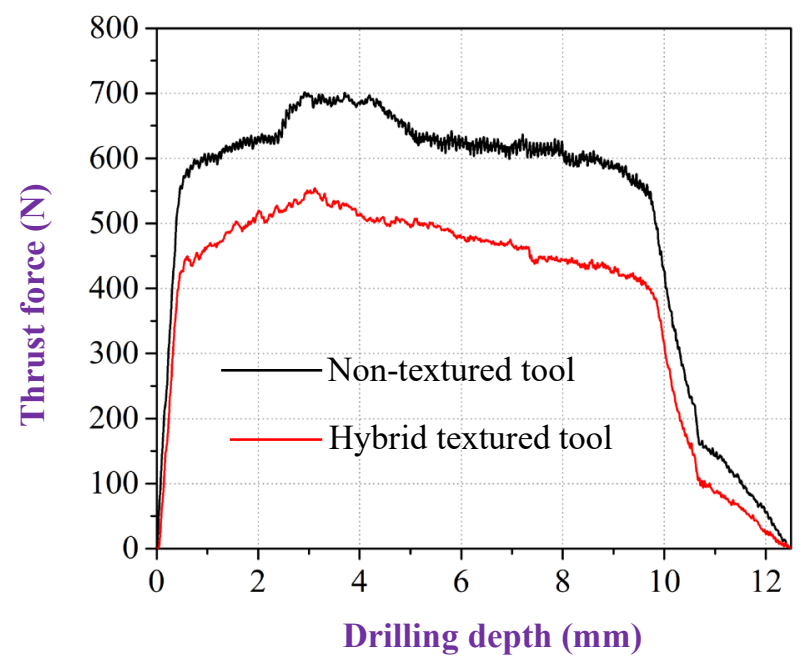

(a) Thrust force

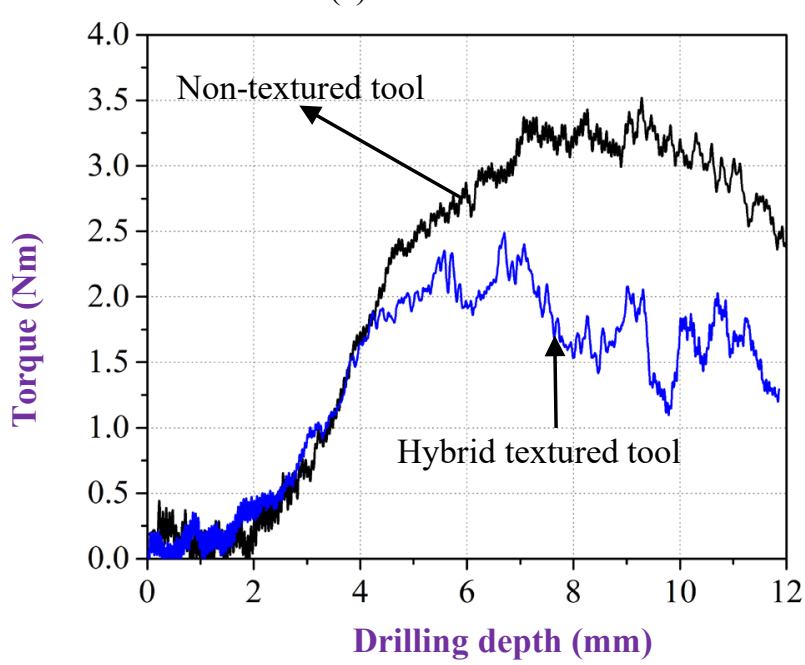

(b) Torque

Fig. 14 Variation in cutting forces during drilling operation 
The variation in thrust force and torque is depicted in the Figure 14. From the drilling experimental results it has been observed that while machining Ti-6Al-4V using Nontextured tool a maximum thrust force of $627 \mathrm{~N}$ was recorded. Whereas for the hybrid textured tool, the thrust force got reduced to $520 \mathrm{~N}$, representing the effectiveness of micro scale textures in minimizing the sling frictional effect at the flute and margin side of the drill tool. Moreover the drilling torque recorded for Non-textured tool was noted to be $2.61 \mathrm{Nm}$ which got reduce to $2.10 \mathrm{Nm}$ for hybrid textured tool. Hence the hybrid textured tool showed a $17.07 \%$ reduction in thrust force and $19.54 \%$ reduction in drilling torque. This shows the effectiveness of the micro texturing principle in improving the cutting performance of the difficult to machine materials like titanium alloys.

\section{Conclusion}

The present research work demonstrated the feasibility of creating hybrid micro scale textures using laser micromachining technique for tribological characteristics. Following are the key findings.

- Numerical modeling validated the underlying mechanism of micro textures in improving the hydrodynamic pressure at the sliding contact regime.

- Tribology experiment revealed the reduction in friction coefficient for micro textured surfaces under both dry and wet sliding conditions. This can be attributed to the mechanism of reduction in contact length, wear debris entrapment, and lubrication enhancement property of the micro-scale textures.

- From the tribology experiments, surfaces with hybrid micro textures were reported to be exhibit lesser friction coefficient showing a net reduction of $32.65 \%$ in comparison with non-textured surfaces.

- Under the dry condition, as the sliding velocity was increased from $0.5 \mathrm{~m} / \mathrm{s}$ to $1 \mathrm{~m} / \mathrm{s}$, a reduction in the coefficient of friction was observed from the tribology experiments. At the lower sliding speed of $0.5 \mathrm{~m} / \mathrm{s}$, coefficient of friction was observed to be 0.43 for the test sample having hybrid textures comparing to the non-textured test sample, which recorded an average coefficient of friction of 0.54 . Hence a net reduction of $20.37 \%$ in friction coefficient in comparison with non-textured surfaces was achieved for the test samples having hybrid textured surfaces.

- Under wet condition also test samples having hybrid textures were observed to be exhibit better friction reduction property which recorded a coefficient of friction of 0.3 at $0.5 \mathrm{~m} / \mathrm{s}$ and 0.23 at $1 \mathrm{~m} / \mathrm{s}$, showing a net reduction of $30.43 \%$ in coefficient of friction in comparison with the test surface without micro scale textures.

- $\quad$ The present work also showed the successful applicability of hybrid micro textures even on the free form surface of the drill tool for improving the cutting performance by minimizing the frictional effect occurring at the machining zone. A total reduction of $17.07 \%$ in thrust force and $19.54 \%$ in drilling torque was achieved by suing hybrid micro textured drill tools.

\section{References}

[1] D. Hamilton, J. Walowit and C. Allen: ASME, J. of Basic Engin., 88, (1966) 177.

[2] D.G. Coblas, A. Fatu, A. Maoui and M. Hajjam: J. Engin. Tribol., 229, (2015) 3.

[3] I. Etsion and E. Sher: Tribol. Int., 42, (2009) 542.

[4] C. Donnet and A. Erdemir: Surf. Coat. Technol., 180181, (2004) 76.

[5] H.A. Jehn: Surf. Coat. Technol., 131, (2000) 433.

[6] S. Lei, S. Devarajan and Z. Chang: J. Mater. Process. Technol., 209, (2009) 1612.

[7] T. Nanbu, N. Ren, Y. Yasuda, D. Zhu and Q.J. Wang: Tribol. Lett., 29, (2008) 241.

[8] D. Zenebe Segu and P. Hwang: Tribol. Int., 91, (2015) 111.

[9] K. Sugioka and Y. Cheng: Appl. Phys. Rev., 1, (2014) 041303-1.

[10]A. Arslan, H.H. Masjuki, M.A. Kalam, M. Varman, R.A. Mufti, M.H. Mosarof, L.S. Khuong and M.M. Quazi: Crit. Rev. Solid State Mater. Sci., 41, (2016) 447.

[11]C.A.A. Rashed, L. Romoli, F. Tantussi, F. Fuso, L. Bertoncini, M. Fiaschi, M. Allegrini and G. Dini: CIRP, J. Manuf. Sci. Tech., 7, (2014) 11.

[12]C.A.A. Rashed, L. Romoli, F. Tantussi, F. Fuso, M. Burgener, G. Cusanelli, M. Allegrini and G. Dini: J. Manuf. Process., 15, (2013) 524.

[13]Z. Wansheng, W. Zhenlong, D. Shichun, C. Guanxin and W. Hongyu: J. Mater. Process. Technol., 120, (2002) 101.

[14]D.S. Patel, V.K. Jain, A. Shrivastava and J. Ramkumar: Int. J. Adv. Manuf. Technol., 100, (2019) 1269.

[15]I. Etsion: Trans. ASME, J. Tribol., 127, (2005) 248.

[16]F. Huber, T. Papke, M. Kerkien, F. Tost, G. Geyer, M. Merklein and M. Schmidt: J. Laser. Appl., 31, (2019) 022318 .

[17]M. Rasch, C. Roider, S. Kohl, J. Straub, N. Maurer, K.Y. Nagulin and M. Schmidt: Opt. Laser Engin., 115, (2019) 179.

[18]K. Sugioka: Nanophotonics., (2017) 393.

[19]K. Sugioka and Y. Cheng: Lab Chip., 12, (2012) 3576.

[20]K. Sugioka and Y. Cheng: Light Sci. Appl., 3, (2014) 1.

[21]J. Heberle, T. Häfner and M. Schmidt: CIRP, Ann., 67, (2018) 197.

[22]L. Romoli, G. Lovicu, C.A.A. Rashed, G. Dini, M. De Sanctis and M. Fiaschi: Pro. CIRP., 33, (2015) 508.

[23]L. Romoli, C.A.A. Rashed, G. Lovicu, G. Dini, F. Tantussi, F. Fuso and M. Fiaschi: CIRP, Ann., 63, (2014) 229.

[24]K. Sugioka, Y. Hanada and K. Midorikawa: Laser Photon. Rev., 4, (2010) 386.

[25]D. Wu, S.-Z. Wu, J. Xu, L.-G. Niu, K. Midorikawa and K. Sugioka: Laser Photon. Rev., 8, (2014) 458.

[26]G.S. Joshi, C. Putignano, C. Gaudiuso, T. Stark, T. Kiedrowski, A. Ancona and G. Carbone: Tribol. Int., 127, (2018) 296.

[27]S.M. Pimenov, B. Jaeggi, B. Neuenschwander, E.V. Zavedeev, O.S. Zilova and M.L. Shupegin: Diam. Relat. Mater., 93, (2019) 42. 
[28]G. Dumitru, B. Lüscher, M. Krack, S. Bruneau, J. Hermann and Y. Gerbig: Int. J. Refr. Metals Hard Mater., 23, (2005) 278.

[29]A.N. Samant and N.B. Dahotre: J. Eur. Ceram. Soc., 29, (2009) 969.

[30]S. Bang and M. Modest: J. Heat Transfer Trans. ASME., 113, (1991) 663.

[31]H. Ki, P.S. Mohanty and J. Mazumder: J. Laser Appl., 14, (2002) 39.

[32]G. Chryssolouris: Springer Sci. \& Business Media., 2013.

[33]K.C. Phillips, H.H. Gandhi, E. Mazur and S.K. Sundaram: Adv. Opt. Photon., 7, (2015) 684-712.

[34]P. Andersson, J. Koskinen, S. Varjus, Y. Gerbig, H. Haefke, S. Georgiou, B. Zhmud and W. Buss: Wear., 262, (2007) 369.

[35]D. Braun, C. Greiner, J. Schneider and P. Gumbsch: Tribol. Int., 77, (2014) 142.
[36]A. Amanov, R. Tsuboi, H. Oe and S. Sasaki: Tribol.Int., 60, (2013) 216-223.

[37]A. Kovalchenko, O. Ajayi, A. Erdemir, G. Fenske and I. Etsion: Tribol. Int., 38, (2005) 219.

[38]I. Etsion and L. Burstein: Tribol. Trans., 39, (1996) 677.

[39]H. Yamakiri, S. Sasaki, T. Kurita and N. Kasashima: Tribol. Int., 44, (2011) 579.

[40]D. Gropper, L. Wang and T.J. Harvey: Tribol. Int., 94, (2016) 509.

[41]A.D. Anoop, A.S. Sekhar, M. Kamaraj and K. Gopinath: Wear., 330-331, (2015) 636.

[42]Y. Kligerman, I. Etsion and A. Shinkarenko: ASME, J. Tribol., 127, (2005) 632.

[43]A. Kovalchenko, O. Ajayi, A. Erdemir, G. Fenske and I. Etsion: Tribol. Trans., 47, (2004) 299.

[44]S. Li and A. Kahraman: Int. J. Fatigue., 33, (2011) 427.

(Received: May 17, 2019, Accepted: November 25, 2019) 